Case report

\title{
A massive hemorrhagic pleural effusion does not exclude the diagnosis of tuberculosis: a case report Charalampos Mermigkis ${ }^{1}$, Antony Kopanakis ${ }^{2}$, Kostas Psathakis ${ }^{1}$, Napoleon Karagiannidis ${ }^{2}$, Manolis Kastanakis ${ }^{2}$, Michael Patentalakis ${ }^{2}$, Anastasia Amfilochiou ${ }^{2}$, Georgios Patentalakis ${ }^{2}$ and Vlassis Polychronopoulos ${ }^{2}$
}

\author{
Addresses: ${ }^{1}$ Department of Pneumonology, Army General Hospital, Athens, Greece \\ ${ }^{2}$ Third Department of Pneumonology, Sismanoglio General District Hospital, Athens, Greece \\ Email: CM* - mermigh@gmail.com; AK - antony_kop@yahoo.gr; KP - kpsazakis@hol.gr; NK - n.karagianidis@windowslive.com; \\ MK - m.kastanakis@yahoo.gr; MP - m.patentalakis@windowslive.com; AA - a.amfilochiou@yahoo.gr; GP - patentalakis@yahoo.gr; \\ VP - vlasispo@hotmail.com \\ * Corresponding author
}

Received: 12 December 2008 Accepted: 6 July 2009 Published: 31 July 2009

Cases Journal 2009, 2:8707 doi: 10.4076/1757-1626-2-8707

This article is available from: http://casesjournal.com/casesjournal/article/view/8707

(C) 2009 Mermigkis et al.; licensee Cases Network Ltd.

This is an Open Access article distributed under the terms of the Creative Commons Attribution License (http://creativecommons.org/licenses/by/3.0), which permits unrestricted use, distribution, and reproduction in any medium, provided the original work is properly cited.

\begin{abstract}
We report a case of an immunocompetent 18-year-old man with a massive hemorrhagic, exudative, lymphocytic pleural effusion. Blind transthoracic pleural biopsy showed granuloma formation, while the pleural fluid culture was positive for Mycobacterium tuberculosis, confirming the diagnosis of primary tuberculous pleuritis. A massive hemorrhagic pleural effusion is extremely rare in tuberculosis, but tuberculosis is a very protean disease and should always be included in the differential diagnosis of pleural effusions
\end{abstract}

\section{Case presentation}

An 18-year-old Greek male, previously healthy, nonsmoker, was referred to our hospital because of chest pain and fever, which had been started about two weeks previously. He had been treated with broad spectrum antibiotics as an outpatient without response. His past medical history was unremarkable.

On admission, he had a temperature of $38^{\circ} \mathrm{C}$, pulse rate 100 beats/min. Physical examination revealed dullness on percussion and absent of breath sounds at the left hemithorax. Laboratory blood tests revealed a reduced hematocrit $(29.5 \%)$ and elevated erythrocyte sedimentation rate $(125 \mathrm{~mm} / \mathrm{h})$ and C-reactive protein $(8.6 \mathrm{mg} / \mathrm{dl})$. Arterial blood gases showed hypoxemia $\left(\mathrm{PO}_{2}=69 \mathrm{mmHg}\right.$ on room air). The rest of the clinical and laboratory examinations were normal. Chest radiography and computed tomography (CT) scan showed a large (more than $2 / 3$ of the left hemithorax was opacified in admission chest radiography), homogenous, non-loculated pleural effusion on the left, with no parenchymal lesions. Flexible bronchoscopy was negative for endobronchial mass, and showed mild edema of the left lower lobe bronchial mucosa, probably due to extrinsic compression. Smears 
and cultures for Mycobacterium tuberculosis taken from bronchial secretions, and bronchoalveolar lavage (BAL) were negative. Cytology and Gram stain of the above specimens were also negative. Diagnostic thoracocentesis revealed an exudative fluid with hemorrhagic appearance (hematocrit $8 \%$, complete cell count $680 / \mu \mathrm{L}$ with $18 \%$ neutrophils and 75\% lymphocytes). Pleural fluid Gram stain, acid-fast bacilli (AFB) smears and cytology were all negative. Pleural fluid adenosine deaminase (ADA) level was $70 \mathrm{IU} / \mathrm{L}$. PPD skin test $(0.1 \mathrm{ml}$ of PPD-RT 23, $2 \mathrm{TU})$ was positive (15 mm induration). Blind needle biopsy of the pleura showed the presence of noncaseating granulomas. Based on these features the diagnosis of tuberculous (TB) pleuritis was established and the patient was started on a 6 month anti-TB regimen with 4 drugs (Isoniazid, Rifampicin, Ethambutol and Pyrazinamide). Clinical improvement was noted gradually. Pleural fluid cultures became positive for M. tuberculosis several weeks later.

\section{Discussion}

To the best of our knowledge this is the first reported case of a massive hemorrhagic pleural effusion due to TB.

$\mathrm{TB}$, the single most frequent infectious cause of death worldwide, is also a major cause of pleural effusion. Primary TB pleuritis most usually manifests as an acute or subacute illness with chest pain, cough, fever or dyspnea being the common symptoms. The effusion is usually an exudate with a lymphocyte predominance and is almost always unilateral and small to moderate in size [1-4].

An acute illness with pleuritic pain, fever and exudative lymphocytic pleural effusion in a young previously healthy man with a positive PPD skin test, in a country with relatively high incidence of infection is strongly suggestive of TB $[1,3]$. However, if the aspirated pleural fluid during thoracentesis is bloody, especially in a large size effusion the diagnosis of TB is considered unlikely [3-5]. In the literature, only four cases of hemorrhagic TB pleuritis have been reported and none of them was massive [6-8]. This has led to the "conventional" knowledge that a hemorrhagic pleural effusion most commonly suggests one of the three following diagnoses: malignant disease, trauma or pulmonary embolization. Less frequent diagnoses for a hemorrhagic pleural effusion may include pneumonia, hematologic disorders or endomitriosis [3].

Currently, the diagnosis of TB pleuritis is practically established when the ratio of lymphocytes to neutrophils in the pleural fluid is $>0.75$ and the ADA is $>70 \mathrm{IU} / \mathrm{L}[2,9]$. The diagnostic yield of pleural fluid culture for $M$. Tuberculosis is generally low and is delayed. Pleural biopsy has the best diagnostic yield for TB pleuritis, but it is rarely necessary when the patient meets the above mentioned criteria [10]. Our patient had a clinical course and pleural fluid characteristics highly suspicious for TB. However, in the presence of a bloody fluid, we preferred to confirm the diagnosis with a pleural biopsy first, before initiating an anti-TB therapy (therapeutic criterion in this case, since the cultures were unknown yet). This case remind us that TB has been recognised as a very protean disease ("one thousand faces disease"), and should always be included in the differential diagnosis of a patient with a lymphocytic pleural exudate, being it hemorrhagic or not, small or massive.

\section{Abbreviations}

$\mathrm{ADA}$, adenosine deaminase; $\mathrm{AFB}$, acid-fast bacilli; $\mathrm{BAL}$, bronchoalveolar lavage; $\mathrm{CT}$, computed tomography; $\mathrm{TB}$, tuberculous.

\section{Competing interests}

The authors declare that they have no competing interests.

\section{Consent}

Written informed consent was obtained from the patient for publication of this case report. A copy of the written consent is available for review by the Editor-in-Chief of this journal.

\section{Authors' contributions}

$\mathrm{CM}$ and $\mathrm{AK}$ wrote the manuscript. NK, MK and AA had the responsibility of the patient during his hospital stay and the patient's data collection. KP had the responsibility of the bibliographic review and references. MP and GP reviewed the manuscript. VP had the overall responsibility of the patient management and the final approval of the manuscript

\section{References}

I. Valdés L, Alvarez D, San José E, Penela P, Valle JM, García-Pazos JM, Suárez J, Pose A: Tuberculous pleurisy: a study of 254 patients. Arch Intern Med 1998, I58:2017-202I.

2. Light RW. Pleural diseases. Philadelphia: Williams and Wilkins; 200I.

3. Fraser R, Pare P: Specific Causes of Pleural Effusion: Mycobacterium Tuberculosis. In Diseases of the Chest. 2nd edition. Edited by Fraser R, Pare P. Philadelphia: W.B. Saunders Company; 1994:869-872.

4. Siebert AF, Haynes J, Middleton R: Tuberculous pleural effussion: 20-year experience. Chest 1991, 99:883-886.

5. Gopi A, Madhavan S, Sharma S, Sahn ST: Diagnosis and Treatment of Tuberculous Pleural Effusion in 2006. Chest 2007, | 3 |:880-889.

6. Murin S, Moritz E: Bilateral Tuberculous Pleural Effusions with markedly Different Characteristics. Chest 1996, I I 0:849-850.

7. Renert WA: Hemorrhagic pleural effusion: an unusual finding in tuberculous pleurisy. Conn Med 1971, 35:549-552.

8. Papamichalopoulos A, Mavrou I, Kostadima E, Trakopoulos G: Bilateral haemorrhagic tuberculous pleuritis in an 8I-yearold female. Int J Tuberc Lung Dis 1999, 3:456.

9. Valdés L, San José E, Alvarez D, Sarandeses A, Pose A, Chomón B, Alvarez-Dobaño JM, Salgueiro M, Rodríguez Suárez JR: Diagnosis of tuberculous pleurisy using the biologic parameters adenosine deaminase, lysozyme, and interferon gamma. Chest 1993, 103:458-465.

10. Escudero-Bueno C, Garcia-Clemente M, Cuesta-Castro B: Cytologic and bacteriologic analyzes of fluid and pleural biopsy with Cope's needle. Arch Intern Med 1990, I 50: I I90-I I 94. 